\title{
Scale-Dependent Community Theory for Streams and Other Linear Habitats
}

\author{
Galen Holt ${ }^{\star}$ and Peter Chesson \\ Department of Ecology and Evolutionary Biology, University of Arizona, Tucson, Arizona 85721 \\ Submitted August 12, 2015; Accepted April 25, 2016; Electronically published July 8, 2016 \\ Online enhancements: appendix.
}

\begin{abstract}
The maintenance of species diversity occurs at the regional scale but depends on interacting processes at the full range of lower scales. Although there is a long history of study of regional diversity as an emergent property, analyses of fully multiscale dynamics are rare. Here, we use scale transition theory for a quantitative analysis of multiscale diversity maintenance with continuous scales of dispersal and environmental variation in space and time. We develop our analysis with a model of a linear habitat, applicable to streams or coastlines, to provide a theoretical foundation for the long-standing interest in environmental variation and dispersal, including downstream drift. We find that the strength of regional coexistence is strongest when local densities and local environmental conditions are strongly correlated. Increasing dispersal and shortening environmental correlations weaken the strength of coexistence regionally and shift the dominant coexistence mechanism from fitness-density covariance to the spatial storage effect, while increasing local diversity. Analysis of the physical and biological determinants of these mechanisms improves understanding of traditional concepts of environmental filters, mass effects, and species sorting. Our results highlight the limitations of the binary distinction between local communities and a species pool and emphasize species coexistence as a problem of multiple scales in space and time.
\end{abstract}

Keywords: coexistence, spatial storage effect, fitness-density covariance, environmental and dispersal scale, stream communities, directional dispersal.

\section{Introduction}

An emerging view of communities is that diversity maintenance fundamentally occurs at the regional scale, where a system is effectively closed to migration on ecological timescales (Chesson 2000a; Ricklefs 2008). In contrast, a more traditional view is that local communities are assembled from a regional species pool and are stabilized by coexistence mechanisms working on the scale of local interactions (Cornell and Harrison 2014). This species pool is often treated as

* Corresponding author; e-mail: gholt@email.arizona.edu.

Am. Nat. 2016. Vol. 188, pp. E59-E73. (C) 2016 by The University of Chicago. 0003-0147/2016/18803-56467\$15.00. All rights reserved. DOI: $10.1086 / 687525$ fixed, but under the emerging perspective, the structure and diversity of the species pool depend on interacting processes functioning at a full range of scales from local to regional (Ricklefs 2008; Chesson 2012). This view focuses on the role of environmental variation and nonequilibrium processes as integral to diversity maintenance (Leibold et al. 2004). These various ideas are applicable to communities generally, including communities in linear habitats such as streams or coastlines, the specific focus of this work.

Although environmental variation has long had a role in the traditional view, it is largely associated with the availability of a species' Hutchinsonian niche on a landscape. Species are assumed to be subject to an environmental filter and so will not be found where their Hutchinsonian niche is absent (Poff 1997; Kraft et al. 2015). On the local scale, species that have passed through the environmental filter are then subject to species sorting (sensu Leibold et al. 2004), where some species are eliminated by competitive exclusion, leaving a subset as a stable local community (HilleRisLambers et al. 2012; Kraft et al. 2015). A modification of this traditional view, mass effects (Shmida and Ellner 1984), accommodates the idea that dispersal from neighboring communities can enrich local diversity beyond that stabilized by the species interactions on the local scale. The emerging view expands on this idea by considering how locally unstable communities can contribute to the emergence of stability on a larger spatial scale.

The emerging view is supported by an assortment of models and concepts with a long history, including ideas about disturbance (Connell 1978; Sousa 1979; Hastings 1980; Caswell and Cohen 1991; Lake 2000); the closely related concepts of metacommunities, patch dynamics, competition-colonization trade-offs, and fugitive species (Hutchinson 1951; Levins 1969; Hanski 1983; Townsend 1989; Tilman 1994); and the effects of environmental variation (Levin 1976; Caswell and Cohen 1995; Amarasekare and Nisbet 2001; Ovaskainen 2002). However, for the most part the emphasis has been on limited numbers of scales or limited model development, without consid- 
eration of the range of scales (Ricklefs 2008). Our aim here is to contribute to the emerging view with a model based on a continuum of scales in space and time and to use it to compare and contrast the concepts from these different perspectives on diversity maintenance. Needed is the ability to understand how regional-scale coexistence is affected by processes on smaller scales, how diversity patterns change with scale, and how they relate to environmental and biological drivers.

Past attempts to assess the roles of multiple scales of environmental variation in community ecology have often been heavily simulation based, and although they produce interesting results that capture important complexity (e.g., Moloney et al. 1992; Lavorel and Chesson 1995; Pacala et al. 1996; Auerbach and Poff 2011), their specificity has limited their impact. Analytical approaches through metacommunity theory have mostly been based on representations of local populations as present or absent. Although such models have provided much insight into the ways spatial scale can affect community dynamics (e.g., Slatkin 1974; Hastings 1980; Hanski 1983; Caswell and Cohen 1991; Tilman 1994; Amarasekare et al. 2004; Muller-Landau 2010), these models do not account for population density in a patch, limiting their ability to represent species interactions, and typically consider only local and regional scales.

Our approach is based on scale transition theory (Chesson et al. 2005; Chesson 2012), which provides a fully quantitative framework ideal for quantifying scale-dependent diversity maintenance mechanisms. Most important, it provides the ability to assess the separate and combined effects of multiple mechanisms acting simultaneously. It does this by providing formulas for mechanism magnitudes. These formulas are given in functional form in terms of components that represent the contributing biological processes (Chesson 2012). These functional forms are powerful because they not only define the mechanisms but also allow their magnitudes to be related to biological and environmental drivers. The analysis of a complex model with scale transition theory uses a hybrid approach where the theory provides the relevant formulas, which are then evaluated numerically. Thus, a complex model can still benefit from the greater generality available from analytical approaches while nevertheless accounting for important complexity that could not normally be considered analytically.

Chesson (2000a) provided the fundamental theory for understanding multiscale species coexistence through scale transition theory. Snyder and Chesson $(2003,2004)$ demonstrated its application to spatially explicit models with the scales of dispersal and competition determined by spatial kernels for a lottery model, with numerical outcomes based on periodic environmental variation in space. The challenge is to extend this work to more realistic environmental variation for a continuum of spatial and temporal scales, to develop a full, multiscaled coexistence theory. Ideally, this theory should show how the absolute and relative importances of different coexistence mechanisms change with the nature and scales of the environmental drivers, dispersal, and species interactions. This theory should show how patterns of abundance and diversity are related to the coexistence mechanisms and their drivers. Finally, it should allow an assessment of the relationships between different perspectives on multiscale diversity maintenance. Although Snyder (2008) addressed the roles of scales of variation for coexistence in an annual plant model, their relationships to specific coexistence mechanisms and scale-dependent diversity patterns remain to be considered.

While our results provide conclusions for general spatially structured communities, they also fill a much-needed gap in the theory of stream communities. The stream and coastal literature emphasizes many of the issues that we raise here, particularly the influence of environmental variation and dispersal on multiscale community structure (e.g., Müller 1954; Vannote et al. 1980; Poff 1997; Mykra et al. 2004; Thompson and Townsend 2006; Heino and Mykra 2008; Brown and Swan 2010; Aiken and Navarrete 2014; Kuglerová et al. 2015). The dominant approaches to these issues are primarily empirical and conceptual, with limited theoretical development. A body of stream theory focuses on population persistence and spatial distributions in the face of biased dispersal downstream (drift; e.g., Speirs and Gurney 2001; Anderson et al. 2005; Pachepsky et al. 2005). Regional-scale species coexistence has been considered in the limited context of $r$ - $K$ tradeoffs postulated to occur along the length of a stream (Levine 2003; Lutscher et al. 2007), but comprehensive multiscaled developments are needed to address the key concerns of stream community ecology. Moreover, such a theory would provide needed guidance for management and restoration of streams, which have struggled to translate small-scale interventions to community outcomes at the reach or watershed scale (Palmer et al. 2014).

Our development is based on a model of species interactions within sites, such as pools, connected by dispersal. In order to study a continuum of environmental scales in space and time, we develop an environmental model allowing independent adjustment of spatial and temporal autocorrelation. Dispersal models are designed to allow assessment of dispersal scales for both symmetric and biased dispersal, addressing the effects of both dispersal distance and downstream drift. Our findings show how community patterns and coexistence mechanisms relate to the underlying environmental and dispersal scales, providing a new scaledependent coexistence theory and illuminating concepts from the traditional view, including species pools, environmental filters, species sorting, mass effects, and neutrality. The results show how the species pool can be stabilized by interactions on lower scales as a consequence of spatial coexistence mechanisms whose relative and absolute contri- 
butions change with the natures of environmental variation and dispersal. Embracing the scale dependence of local composition and stabilization of diversity in the species pool provides the foundation for a more robust quantitative spatialcommunity ecology.

\section{Model of Stream Communities}

We model streams as series of connected sites. We assume that the physical environment varies along the length of the stream, reflecting features such as contrasts between pools and riffles, water chemistry, and substrate. Likewise, the environmental conditions at each site vary in time as well, as a result of such factors as discharge variability, bedform changes, and turbidity or other changes to water quality. However, the physical environment is not explicitly modeled. Instead, we represent the response of each species to the varying environmental conditions occurring along the stream with the variable $E_{j, x}(t)$, where $j$ refers to species, $x$ the spatial location, and $t$ time. The environmental response, $E_{j, x}(t)$, is the environmentally dependent fitness of an individual before competition is accounted for and before mortality during dispersal from site $x$. The fitness overall, $\lambda_{j, x}(t)$, is then

$$
\lambda_{j, x}(t)=S_{x} E_{j, x}(t) e^{-C_{j, x}(t)},
$$

where $C_{j, x}(t)$ is the magnitude of competition experienced by species $j$ for the specific location and time and $S_{x}$ is the sitespecific dispersal survival probability that accounts for the average mortality during dispersal from site $x$ during the period from $t$ to $t+1$. For simplicity, we have assumed here that $S_{x}$ does not vary with species and time.

We assume that the overall magnitude of competition is a linear function of the total fitness, before accounting for competition, of all individuals at the site. Thus, it is linear in the densities $N_{l, x}(t)(l=1, \ldots, n)$ of the organisms and satisfies the formula

$$
C_{j, x}(t)=\alpha \sum_{l} E_{l, x}(t) N_{l, x}(t)
$$

where $\alpha$ is a competition coefficient. Our rationale for having competition be a function of the Es is that more favorable environmental conditions should lead to higher levels of foraging activity, which should proportionately draw down resources. This assumption is the basis of many models of the role of environmental variation in species coexistence, where further justifications may be found (Chesson 1994, 2000a, $2000 b, 2012$ ). We expect that results from this model will be qualitatively similar to those from a broad class of models, as long as they meet two critical criteria: species performance is dependent on variable environmental conditions, and those conditions mediate density-dependent interactions (Chesson 2000a, 2012).
The dynamics of the model work as follows: the density $N_{j, x}(t)$ of organisms of species $j$ present in site $x$ at time $t$, through reproduction and survival, gives rise to $E_{j, x}(t) e^{-C_{j, x}(t)}$ $N_{j, x}(t)$ organisms during the interval from $t$ to $t+1$. By $t+$ 1 , these organisms have dispersed to other sites or remained in $x$, according to a dispersal kernel, $k(z)$, which is a function giving the probability of dispersal from $x$ to $x+z$. For simplicity, we assume that the kernel $k(z)$ is time, location, and species independent. To model different upstream and downstream dispersals, allowing for $\mathrm{drift}$, we introduce a parameter $\delta$ for the probability that a dispersing organism goes downstream and then use a negative binomial distribution for the dispersal distance, which might be 0 ("Modeling Details" in the appendix, available online). For the results presented here, we assume that organisms that disperse beyond the mouth of the stream are lost (washout), while those that attempt to disperse beyond the headwaters reflect back downstream. The resulting spatially variable dispersal mortality leads to the site-specific dispersal survival fraction, $S_{x}$, of equation (1).

The environmental response and environmental scales. We model the environmental responses of the different species as independent lognormal variables. Thus,

$$
E_{j, x}(t)=e^{U_{j, x}(t)},
$$

where $U_{j, x}(t)$ is a normal random variable with mean $m_{j}$ and variance $s_{j}^{2}$. Although we assume that the species have independent environmental responses, it is critically important that these environmental responses are correlated in time and space within a species to define the spatial and temporal structure of the environment. In the simplest case, we assume that the environment varies only in space, that is, only with $x$, and thus can be written $E_{j, x}$. To create spatial structure, we generate the variables $U_{j, x}$, where $x=0,1, \ldots$, as a stationary autoregressive process. As described in "Modeling Details," the autocorrelation function of $E$, that is, the correlation between $E_{j, x}$ and $E_{j, x+d}$ as a function of the separation of the sites in space, $d$, can be expressed approximately as a negative exponential ("Modeling Details"),

$$
A(d)=e^{-d / \tau},
$$

where the constant $\tau$ defines the characteristic length scale at which the environmental response changes, known as the correlation length (Janke 1996).

To model environmental variation in both space and time, we generate $U_{j, x}(t)$ with a two-parameter autoregressive process ("Modeling Details"), which introduces both spatial and temporal correlation lengths. Different correlation lengths correspond to different sorts of environmental variation. Short correlation lengths represent rapid environmental change in space or time, such as pool-riffle sequences or year-to-year differences in the weather. Longer correlation lengths repre- 
sent larger-scale changes, such as water chemistry along the stream or longer-term changes to climate. However, such interpretations should be made with caution; the correspondence of correlation lengths to ecological factors is meaningful only relative to patch and stream size. Unless noted otherwise, we model species' environmental responses as independent. Thus, environmental correlations do not favor one species over the other, on average. Another length scale is important, but it emerges not from the structure of the environment but from the dispersal kernel and is simply the mean dispersal distance. However, because of the downstream bias due to drift, the parameter $\delta$ for the downstream dispersal fraction is needed too.

Figure 1 illustrates spatial patterns of abundance along the length of a stream given by simulations of the model for the two-species case. We present spatial distributions for different scales of dispersal relative to environmental variation, referred to as "relative dispersal" (mean dispersal distance/spatial correlation length). Figure 1 illustrates these distributions for both pure spatial variation in the environmental responses, where an equilibrium spatial pattern occurs (left column), and spatiotemporal variation in the environmental responses, where the spatial pattern continuously changes (middle and right columns; temporal correlation length $=1.0$ ). With no dispersal (row 2), the species cannot coexist at a site, which is taken by the species with the higher environmental response in the case of pure spatial variation. In the case of spatiotemporal variation, this process of site-level competitive exclusion proceeds slowly, but each site is eventually taken by the species with the greater long-run mean environmental response. Hence, with no dispersal, there is no appreciable change over time in the dominant species at a site, even though the environment has changed substantially between the middle and right columns. As relative dispersal increases (rows 3 and 4), populations spread more evenly over the stream, but this effect is much stronger with spatiotemporal variation. Indeed, with spatiotemporal variation, only a small amount of dispersal is needed for both species to be found at appreciable densities at all sites. Drift (bottom row) shifts the spatial distributions downstream but has relatively minor effects on site-level diversity.

To understand the dependence of diversity on scale, we calculate the average Shannon diversity for sections of a stream of a specific length (window length) and see how diversity changes with window length for different levels of dispersal and different temporal autocorrelation lengths (fig. 2). For the smallest spatial scale (a single site), local diversity increases with dispersal distance and the rapidity of temporal environmental change. Moreover, a greater proportion of the stream must be considered to capture the diversity of the entire stream when species are highly segregated because of low relative dispersal or slow temporal environmental change. For a full understanding of the significance of such patterns, we need to understand how regional-scale coexistence mechanisms relate to them.

\section{Regional Dynamics and Coexistence}

By definition, stable competitive coexistence occurs when species recover after perturbation to low density (hereafter "invaders") in the presence of their unperturbed competitors (hereafter "residents"; Turelli 1978; Chesson and Ellner 1989; Schreiber 2012). This recovery rate must be studied at the scale at which the community is effectively closed, as that is the scale that captures all processes that can appreciably affect the recovery rate (Chesson 2000a). In our model, as in nature, environmental conditions vary between locations, and dispersal connects the dynamics of sites with different environmental conditions. Thus, we analyze the recovery rate at the scale of the whole stream, which integrates over the joint effects of site-scale interactions and dispersal on regional dynamics.

Coexistence is quantified by measuring the rate of recovery for each species in a multispecies community. The invasion rate is measured as the multiplicative growth rate $\tilde{\lambda}_{i}$, which can be thought of as the landscape-scale fitness (Chesson 2012), and defines growth from low density (the invader state) as

$$
\bar{N}_{i}(t+1)=\tilde{\lambda}_{i} \bar{N}_{i}(t) .
$$

Here, $\bar{N}_{i}(t)$ is the average density of species $i$ in the region. A value of $\tilde{\lambda}_{i}$ greater than 1 means that species $i$ recovers from low density. Conversely, a value less than 1 means that it cannot recover. Given the heterogeneity between sites, the fitnesses of individual organisms differ from $\tilde{\lambda}_{i}$. Instead, $\tilde{\lambda}_{i}$ is the outcome of an integration over the full set of heterogeneous conditions that individuals encounter.

Scale transition theory provides the mathematical framework necessary for this integration and takes account of species interactions to predict dynamics regionally (Chesson 2008, 2012). For coexistence studies, scale transition theory allows the contributions from different coexistence mechanisms to be quantified. For example, in the model used here, scale transition theory allows us to partition the low-density (invader) recovery rate into contributions from two coexistence mechanisms, the spatial storage effect $\overline{\Delta I}$ and fitnessdensity covariance $\overline{\Delta \kappa}$, with their contributions to recovery from low density given as

$$
\tilde{\lambda}_{i}-1=\xi_{i}+\overline{\Delta I}+\overline{\Delta \kappa}
$$

("Derivation of Coexistence Mechanisms" in the appendix) Here the $\xi_{i}$ represent average fitness differences between species. This quantity compares the average fitness of species $i$ as invader with the average for all of its competitors, also in the invader state (Chesson 2003). These average fitness 


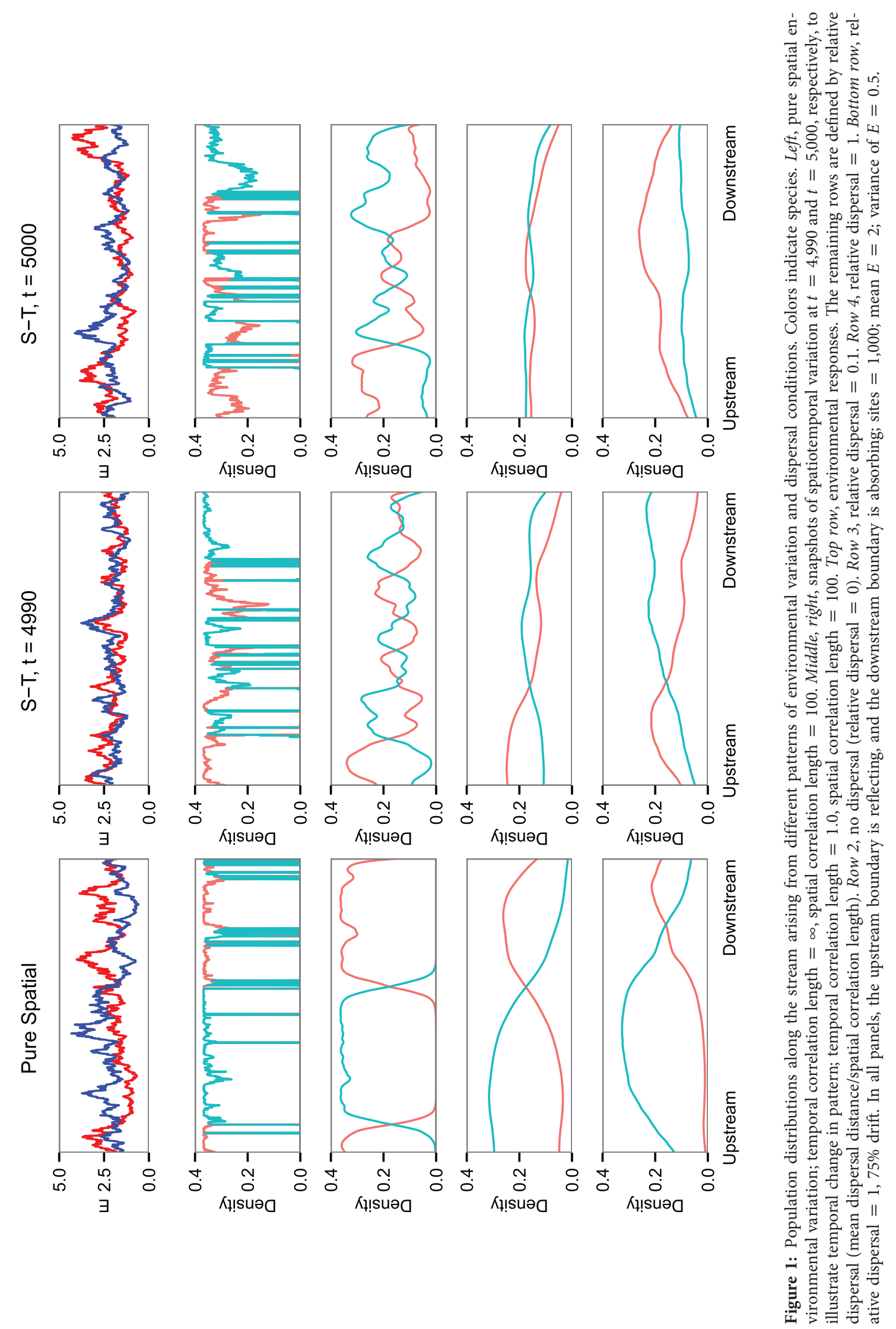

E63

This content downloaded from 150.135.118.172 on October 10, 2016 13:08:16 PM 


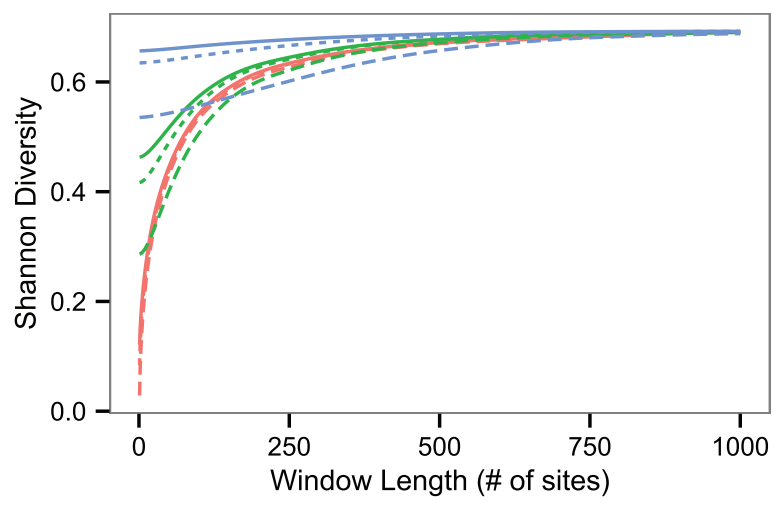

Figure 2: Shannon diversity at different spatial scales (window lengths) dependent on dispersal and environmental scale. Relative dispersal is 0 (red lines), 0.1 (green lines), or 1.0 (blue lines). Temporal correlation lengths $=0.1$ (solid lines), 1 (dotted lines), or 10 (dashed lines). Shannon diversity at a window length $l$ is found by calculating the Shannon diversity of the total community contained in $l$ contiguous sites; the resulting diversities for each group of $l$ sites contained in the stream are averaged to yield the average Shannon diversity at that window length. A window with a size of 1 is a single site, while a window with a size of 1,000 is the entire stream, capturing regional diversity.

differences sum to 0 , and in the absence of coexistence mechanisms, species with negative $\xi$ values would not recover from low density. In this model, however, $\overline{\Delta I}$ and $\overline{\Delta \kappa}$ are often positive and if sufficiently large can outweigh negative average fitness differences to give positive recovery rates for all species, and hence stable coexistence. The combined strength of the coexistence mechanisms determines the maximum av- erage fitness difference at which coexistence is stable. We therefore refer to the combined strengths of fitness-density covariance and the spatial storage effect as the total strength of coexistence stabilization. The strength of coexistence is important beyond determining stability. Provided that coexistence is stable, increasing the strength of coexistence increases the relative abundance of species with lower average fitness (fig. 3A). Another way of understanding such stability is robustness to long-term environmental change. Environments in nature are generally subject to slow long-term environmental change, which is rarely considered in models. Such change is likely to favor one species over another, increasing average fitness differences over time. Increased mechanism strength lengthens the persistence time of the weaker species under such environmental change (fig. 3B).

The spatial storage effect and fitness-density covariance coexistence mechanisms are defined by mathematical expressions that quantify ecologically meaningful relationships (Chesson 2012). For our model, these are given in table 1 . The functional forms of these mathematical expressions provide critical mechanistic understanding of regional-scale coexistence. The storage effect measures the regional-scale effects of separating the physiological activity of different species in space (box 1), while fitness-density covariance measures the regional-scale outcomes of separation of species numerically (box 2). These general features define the mechanisms and are not specific to any particular model (Chesson $2000 a$ ). The analytical formulas defined by scale transition theory facilitate the use of a hybrid approach where the formulas provide mechanistic understanding while their values are calculated numerically by simulation for specific models.
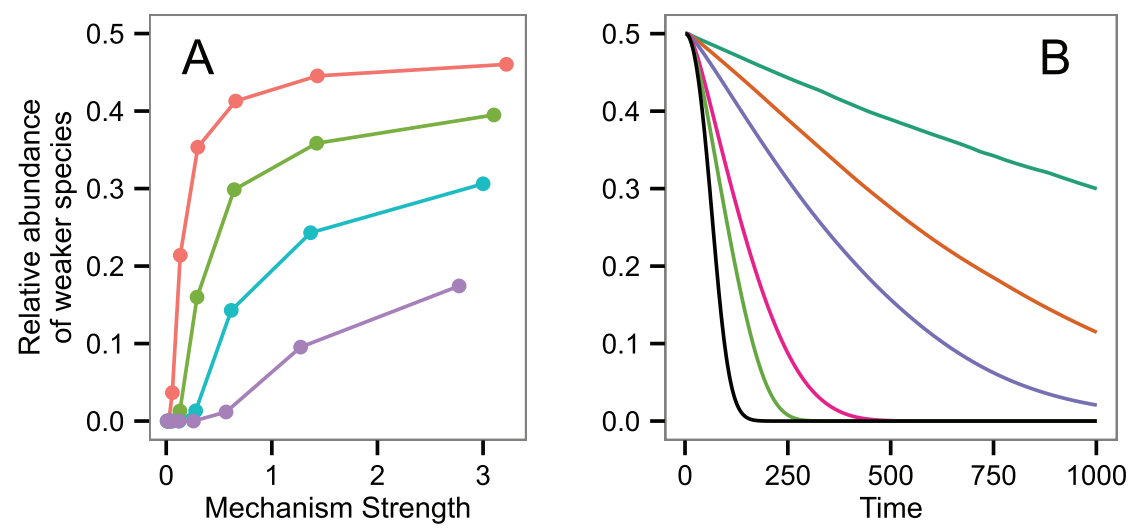

Figure 3: Effect of mechanism strength and average fitness differences on relative abundance. $A$, No long-term environmental change; relative abundance against mechanism strength for different average fitness differences. Average fitness difference ( $\xi$ in table 1$)$ is 0.1 (red line), 0.25 (green line), 0.5 (blue line), or 1 (purple line). Mechanism strength is varied by varying the common variance of the environmental responses, $E_{j}$. $B$, Long-term environmental change; average fitness difference ( $\xi$ in table 1$)$ increases from 0 at the rate of 0.001 per unit time. Trend is linear, not driven by temporal correlation length. Mechanism strengths: 3.0 (dark green line), 2.0 (orange line), 0.84 (purple line), 0.15 (magenta line), 0.08 (olive line), 0 (black line). Mechanism strength is varied by varying relative dispersal (parameters given in "Modeling Details" in the appendix, available online). 
Table 1: Components of invader fitness at the landscape scale

\begin{tabular}{ll}
\hline Mechanism & \multicolumn{1}{c}{ Formula } \\
\hline $\begin{array}{l}\xi_{i} \text { (average fitness } \\
\text { difference) }\end{array}$ & $\left(\bar{E}_{i}^{x}-{\overline{E_{j}}}^{j \neq i}\right) / \overline{\bar{E}}$ \\
$\overline{\Delta I}$ (spatial storage \\
$\begin{array}{l}\text { effect) } \\
\overline{\Delta K} \text { (fitness-density } \\
\text { covariance) }\end{array}$ & $\overline{\left(\overline{\operatorname{Cov}_{x}\left(\mathcal{E}_{j, x} \mathcal{C}_{x}^{-i}\right)}{ }^{i \neq j}-\operatorname{Cov}_{x}\left(\mathcal{E}_{j, x} \mathcal{C}_{x}^{-j}\right)\right)^{j}}$ \\
\hline
\end{tabular}

Note: Subscript $i$ : invader; superscript $-j$ : in the absence of species $j$ (species $j$ is invader); superscript $i \neq j$ : average over all other species in turn as invader, with species $j$ as resident; $\mathcal{E}_{j, x}$ : effect of $S_{x} E_{j, x}$, expressed in units of fitness; $\mathcal{C}_{j, x}$ : effect of $C_{j, x}$ expressed in units of fitness; $\nu_{j, x}$ : relative density of species $j$ at site $x\left(N_{j, x} / \bar{N}_{j}{ }^{x}\right)$. Note that $\nu_{j, x}$ is a within-species, between-sites measure (box 2), $\overline{\bar{E}}$ is the mean environmental response over sites and species. Covariances are taken within species over space. Derivations of these equations for our model are given in "Derivation of Coexistence Mechanisms" in the appendix, available online.

This approach allows a robust, mechanistic investigation of how regional diversity maintenance depends on complex interactions between environmental heterogeneity, dispersal distance, and downstream drift. Since any particular simulation run reflects a single realization of the environmental variation, the results presented here were obtained by repeating simulations until standard errors were negligible. All simulations were run for at least 1,000 generations to ensure that dynamics were independent of initial conditions.

\section{Results}

Presentation of the results is simplified by the finding that the ratio of mean dispersal distance to spatial correlation length of the environmental response is a fundamental scaling relationship determining total coexistence strength, partitioning of coexistence between mechanisms, and diversity patterns. Thus, the separate scales of dispersal and environmental variation do not affect the results when this ratio remains fixed ("Relative Dispersal as a Scaling Relationship" in the appendix), and we present our results in terms of this scale of "relative dispersal" (mean dispersal distance/spatial correlation length). While our model can be extended to continuous space, the discrete nature of the simulations does cause minor deviations for very small absolute scales of dispersal and environmental variation ("Relative Dispersal as a Scaling Relationship"). Since the relative scales are the important scales, we use large-enough absolute scales of dispersal and environmental variation to avoid issues of discreteness in the simulations. The scale of environmental variation relative to the number of sites in the stream determines the total amount of environmental heterogeneity. In order to fix the total amount of environmental variation as the spatial correlation length changes, we make the stream length proportional to spatial correlation length. Except where indicated otherwise, our streams are 100 times as long as the spatial correlation length, which is long enough to ensure that

\section{Box 1: Spatial storage effect}

The storage effect quantifies how the spatial separation of physiological activity can promote coexistence, illustrated in box figure 1 . When the environment at a site is favorable for residents, their resulting high physiological activity (resident environmental response, blue line) increases per-capita demand for resources at that location, increasing competition (black line). However, high physiological activity of invaders (invader environmental response, red line) does not increase competition, because they have negligible density and thus do not draw down resources. It follows that invaders are not limited by competition in their favored locations unless residents are also favored there. The extent to which favorable environmental conditions are limited by competition is quantified by the covariance between environmental response and competition (covEC). When covEC is lower for the invader than for the resident, residents limit themselves more than they limit invaders on average over the region, and coexistence is promoted by the spatial storage effect. This resident-invader comparison of covEC is expressed mathematically in table 1.

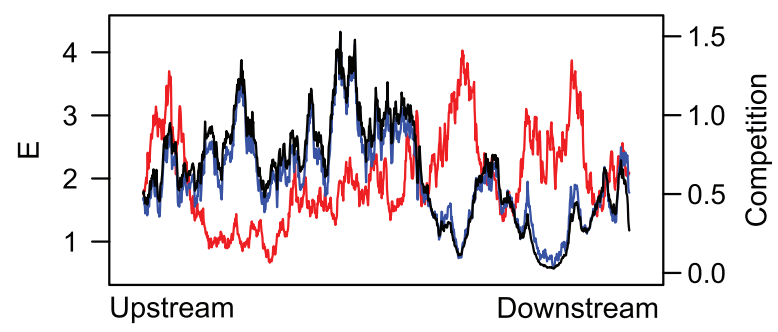

Box Figure 1: Environmental and competitive responses for an invader and a resident. The blue line is for resident environmental response, the red line is for invader environmental response, and the black line is for competition. Parameters (and $E$ distributions) as in figure 1, left column, middle row. 


\section{Box 2: Fitness-density covariance}

Fitness-density covariance, illustrated in box figure 2, quantifies how numerical concentrations affect coexistence. Intuitively, the average fitness of all individuals in the region increases when organisms concentrate in areas of high fitness. How strongly organisms are concentrated in areas of high fitness is measured by the covariance (covFD) between fitness at a site, $\lambda_{j, x}(t)$, and the site-scale relative density of the species, $\nu_{j, x}(t)$. Relative density is the ratio of the density of species $j$ at site $x$ to its average density in the stream, $\nu_{j, x}(t)=$ $N_{j, x}(t) / \bar{N}_{j}(t)$. Note that $\nu_{j, x}(t)$ is a within-species, between-site measure, not the abundance of one species relative to other species ("relative abundance"). From this definition, relative density averages to 1 over space, regardless of the species' absolute density. Thus, a value of 2 still means that a local site has twice the average density, even if that average density is vanishingly small. This fact remains true in the limit as average density approaches 0 and the species is in the invader state. Since residents generate competition, both fitness at a site (brown line in box fig. 2) and density are limited, even in sites where the species has a high environmental response. These limitations mean that there is relatively small variation in both relative density (orange line) and fitness (brown line) along the stream and hence low $\operatorname{covFD}$ (formally, $\operatorname{Cov}_{x}\left(\lambda_{j, x}, \nu_{j, x}\right)$ ). In contrast, invader fitness (purple line) can be much greater than 1 at sites with high $E_{i}$ (especially if resident densities are low) because invaders do not generate appreciable competition. Locations of high invader fitness generate high relative density (green line) because organisms build up in abundance over time in areas of high fitness if there is some local retention, leading to high covFD. This covFD difference between invaders and residents is quantified mathematically as the mechanism termed "fitness-density covariance" (table 1).

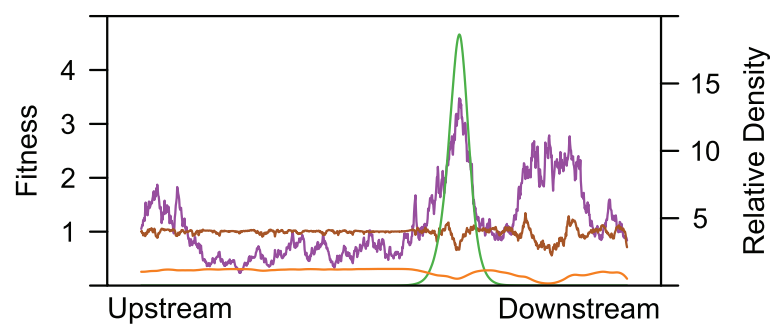

Box Figure 2: Fitness and relative density for an invader and a resident. The purple line is for invader fitness, the brown line is for resident fitness, the green line is for invader relative density, and the orange line is for resident relative density. Parameters (and $E$ distributions) as in figure 1 , left column, middle row.

we capture a representative sample of the environmental responses.

We begin by analyzing purely spatial environmental variation (Chesson 1985). Thus, the spatial environmental pattern along the stream does not change with time. Total stabilization of coexistence (fitness-density covariance + spatial storage effect) at the regional scale is highest when dispersal is short relative to spatial environmental variation (red line in fig. $4 A$ ) and decreases as relative dispersal increases. This shift from high to low coexistence strength regionally coincides with a shift from low to high Shannon diversity at small spatial scales, as can be seen in figures 1 and 2 . The changes in the total strength of coexistence are primarily driven by changes in the strength of fitness-density covariance (blue line in fig. $4 A$ ). When relative dispersal is low, organisms tend to remain in environmental conditions similar to those of their birth. This retention means that invaders concentrate in sites where they have a favorable environmental response, generating strong fitness-density covariance. As relative dispersal increases, organisms disperse to locations with more varied environmental conditions and lower fitness on average, undermining fitness-density covariance. Furthermore, this increased dispersal increases the importance of washout at the mouth of the stream, although this outcome typically has a negligible effect on the overall strength of coexistence (fig. 5; "Detailed Examinations of Coexistence Mechanisms" in the appendix).

While the storage effect (green line in fig. 4A) is often weaker than fitness-density covariance, it is nearly unaffected by the relative scales of dispersal and spatial environmental variation, and its relative contribution to the stabilization of regional coexistence increases as relative dispersal undermines fitness-density covariance (fig. $4 B$ ). As the spatial storage effect depends on the spatial separation of physiological activity, it is primarily driven by the overall amount of variation in environmental responses (box 1). The spatial separation of density, which so strongly influences fitness-density covariance, makes only a small contribution to the spatial storage effect ("Detailed Examinations of Coexistence Mechanisms"). In an extreme case, uniform global dispersal completely prevents numerical buildup over time at any location, eliminating fitness-density covariance while the storage effect remains relatively unchanged and capable of stabilizing coexistence ("Detailed Examinations of Coexistence Mechanisms").

Downstream drift weakens the total stabilization of coexistence, but its effect is generally weaker than the effect of 

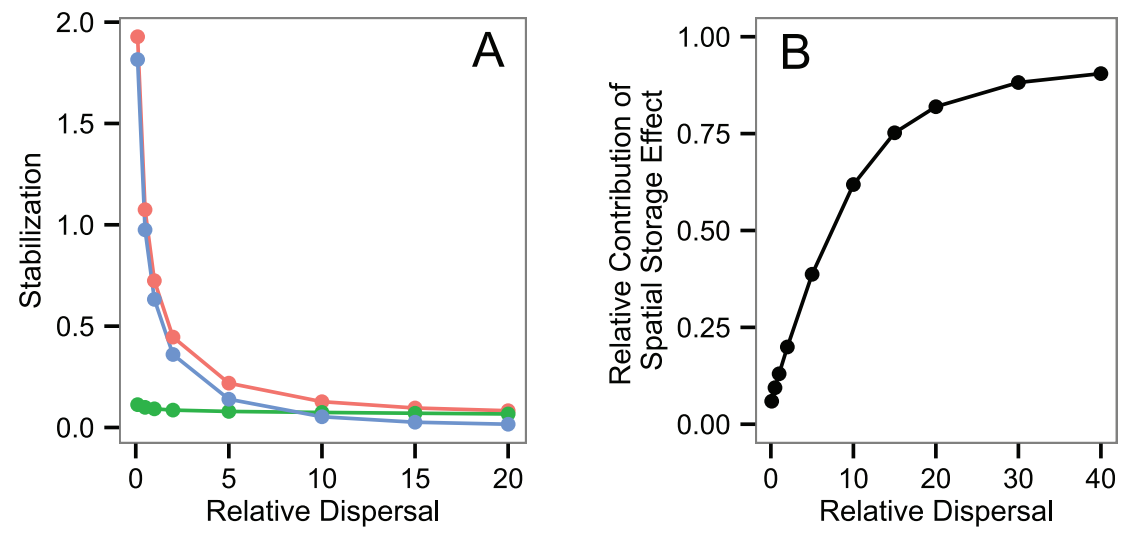

Figure 4: $A$, Total coexistence stabilization and contributions of fitness-density covariance and spatial storage effect with spatial environmental variation. The red line is for total stabilization, the blue line is for fitness-density covariance, and the green line is for the spatial storage effect. $B$, Proportion of total stabilization contributed by the spatial storage effect. "Relative dispersal" is the ratio of mean distance dispersed to the spatial correlation length of the environmental responses.

relative dispersal (fig. 5). Like overall dispersal levels and temporal correlation structure, drift has a much larger effect on the strength of fitness-density covariance than on the strength of the storage effect, causing the storage effect to increase in importance relative to fitness-density covariance as drift increases (fig. 5). Much like long-distance dispersal, drift weakens the ability of organisms to remain in favorable environmental conditions. Drift accentuates this effect because areas of highest relative density are biased downstream from the locations of highest fitness, rather than spread symmetrically about these high-fitness locations ("Detailed Examinations of Coexistence Mechanisms"). Moreover, both high relative dispersal and high drift increase the impor- tance of boundary conditions for regional dynamics, with mortality from washout at the mouth of the stream being particularly important. High drift with high relative dispersal can lead to negative fitness-density covariance, because organisms tend to concentrate in downstream locations, where they have low fitness from washout. In figure 5, this phenomenon leads to a relative contribution of the storage effect greater than 1, meaning that coexistence would be stronger on the basis of the storage effect alone. To examine the importance of these boundary effects, we compare to a situation where the stream is a loop: dispersal from the mouth moves organisms to the headwaters. The closeness of the solid and dashed lines in figure 5 shows that boundary effects tend to
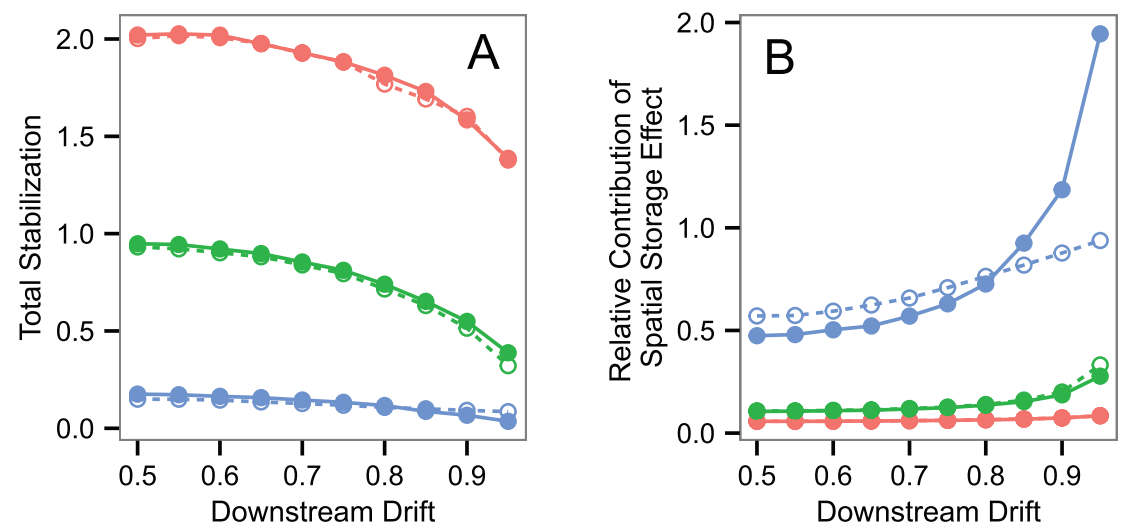

Figure 5: Mechanism strengths depend on relative dispersal and drift. Downstream drift is the proportion of dispersers that disperse downstream; 0.5 indicates symmetric dispersal. Relative dispersal is 0.1 (red line), 1 (green line), or 10 (blue line). Solid lines and filled circles: the mouth of the stream is an absorbing boundary; dashed lines and open circles: the stream is a loop (dispersal from the mouth goes to headwaters). See "Detailed Examinations of Coexistence Mechanisms" in the appendix, available online, for separate strengths of each mechanism. 

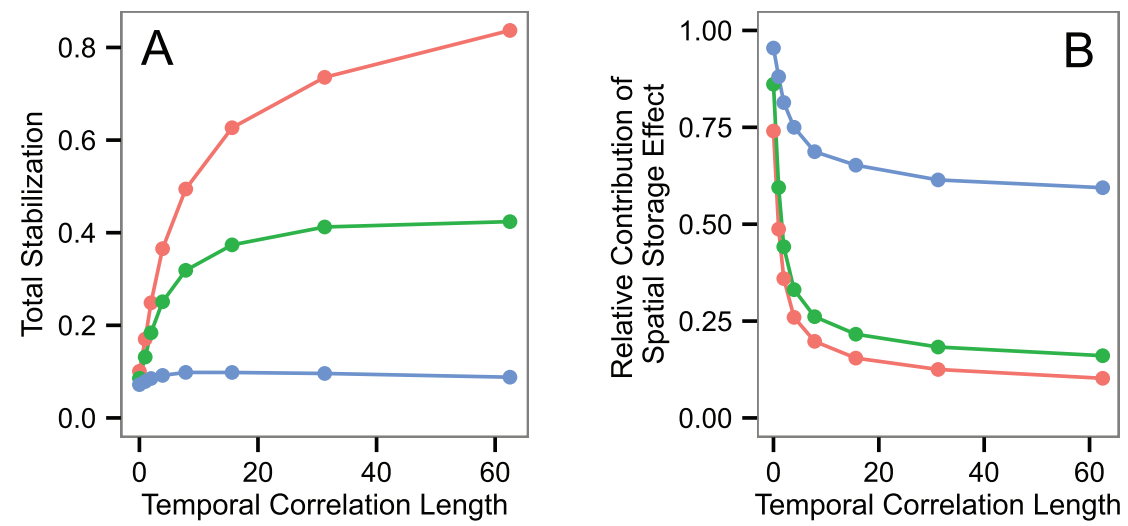

Figure 6: Stabilization of coexistence with spatiotemporal environmental variation. Relative dispersal (mean dispersal distance/spatial correlation length of environment) is 0.1 (red lines), 1 (green lines), or 10 (blue lines). With zero temporal correlation length, environmental conditions at each site are independent between time steps. As temporal correlation lengths approach infinity, environmental variation approaches pure spatial variation.

be less important than the asymmetric shifts in abundance away from natal habitat, except where dispersal and drift are great enough that organisms concentrate in areas of high dispersal mortality.

Similar to pure spatial variation, spatiotemporal variation often yields a fitness-density covariance much larger than the spatial storage effect, but fitness-density covariance is undermined when buildup in favorable locations is limited. With spatiotemporal variation, this limitation need not be due to excess dispersal from favorable conditions but is due to rapid changes in environmental conditions that occur with low temporal correlation length (fig. 6). Not only does spatiotemporal variation increase diversity at smaller spatial scales relative to pure spatial variation (fig. 2), it also reduces the correlation between abundances and current environmental responses (figs. 1, 7; "Pattern Analysis" in the appendix). Although organisms may build up at a site as a result of past conditions, fitness at a site depends on current environmental conditions, and so low temporal correlation lengths undermine fitness-density covariance. However, fitnessdensity covariance rapidly strengthens as temporal correlations increase, especially when relative dispersal is low. Like high relative dispersal, low temporal correlation length hardly affects the spatial storage effect. Thus, the relative importance of the spatial storage effect increases as the temporal correlation length of environmental variation decreases (fig. 6).

Like the strength of coexistence, patterns of site-level diversity along the stream depend on relative dispersal and
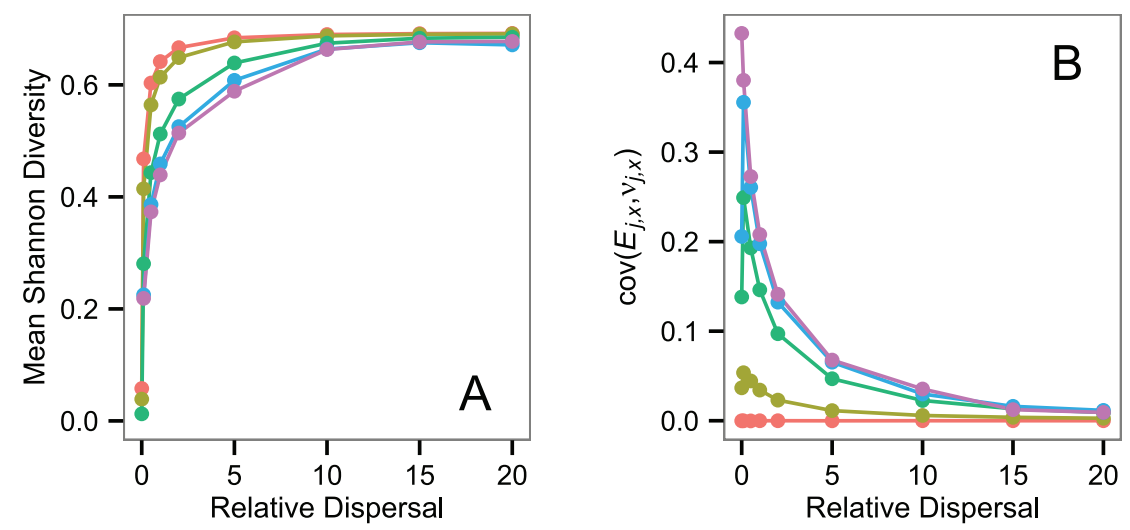

Figure 7: Community composition at the site scale depends on relative dispersal and temporal correlation length. A, Average Shannon diversity of sites. $B$, Association between environment and density, quantified as $\operatorname{Cov}\left(E_{j, x}, v_{j, x}\right)$. Temporal correlation lengths: 0 (red line; sites are independent in time), 1 (gold line), 10 (green line), 100 (blue line); the purple line is for pure spatial variation, no change with time. Coexistence is stable regionally in all cases; species cannot contribute to diversity if they cannot persist regionally. Although calculated at the scale of a single site, the smooth changes in Shannon diversity seen in figure 2 suggest that these patterns would change smoothly with changes in window length. 
the temporal correlation length of the environmental responses (fig. 7). The Shannon diversity of single sites, averaged over the stream, increases with increasing relative dispersal. This occurs for the same reason that fitness-density covariance declines: higher relative dispersal distributes organisms more evenly over the stream. Likewise, increasing relative dispersal reduces the association between the environment and relative densities at a site. These patterns of sitescale diversity apply under stable regional coexistence. In contrast, if conditions do not permit a species to persist regionally, it cannot contribute to diversity at any scale. Short temporal correlations yield high Shannon diversity at single sites and low $\operatorname{Cov}\left(E_{j, x}, \nu_{j, x}\right)$ because environmental conditions change quickly enough to prevent any single species from dominating or concentrating in a site. As environmental conditions become more stable through time, species are more likely to numerically concentrate in sites where they have a high environmental response, increasing $\operatorname{Cov}\left(E_{j, x}, \nu_{j, x}\right)$ and decreasing the average diversity of single sites. Only a small amount of temporal correlation yields large increases in $\operatorname{Cov}\left(E_{j, x}, \nu_{j, x}\right)$ (fig. 7), similar to the increases in fitness-density covariance seen in figure 6.

\section{Discussion}

\section{Community Concepts}

Our work challenges the common conceptual distinction in ecology between the local community and the species pool. Dispersal means that every location is affected by its neighbors, and there are no well-defined local communities. Choosing a particular scale on which to define a local community is an arbitrary choice because there is no local scale on which species distributions or combinations of species distributions have clear boundaries (fig. 1). Of most importance, species interactions change continuously with scale. This fact leads to the scale dependence of the two key coexistence mechanisms, fitness-density covariance and the spatial storage effect, that have emerged in this study.

Because the variation in the physical environment is critical for diversity maintenance in our model, a strong relationship between a species' local abundance and environmental favorability might be expected. Indeed, such a relationship is ound when fitness-density covariance is the dominant mechanism of diversity maintenance. However, a weak relationship or the absence of a relationship is possible with the spatial storage effect. The reason is that the spatial storage effect reflects partitioning the environment at the level of the physiology of the individuals, that is, at the level of individual responses to the environment. If the environment is changing over time or dispersal is large, then individual-level responses to the environment need not be reflected by strong abundance-environment relationships (figs. 1, 7). Under these conditions, fitness- density covariance is minor and the spatial storage effect is the dominant coexistence mechanism (figs. 4, 6). Thus, the presence of all species at high abundance at all local sites, with little relationship between environmental conditions and species densities, is fully consistent with the stabilization of diversity by the spatial storage effect. Although such spatial patterns are often regarded as indicating neutrality and a lack of niche-based processes (e.g., Gilbert and Lechowicz 2004; Cottenie 2005; Thompson and Townsend 2006; Heino and Mykra 2008), our work shows that such conclusions cannot be drawn.

High local abundance of a species where it is not favored environmentally has been discussed in the literature in terms of mass effects and source-sink dynamics (Shmida and Ellner 1984; Pulliam 1988; Leibold et al. 2004). The concept of mass effects makes the implicit assumption that a local community can be well defined yet highly dependent on immigration. Mass effects are often viewed as an alternative to a coexistence mechanism. Instead, our developments imply they should be viewed from the perspective of a larger spatial scale. The conditions for coexistence may not be satisfied within individual units at any local spatial scale but are collectively satisfied on larger scales by the variation in the environment from unit to unit on the local scale, as we see here. Thus, local diversity arising from dispersal should be viewed not as the absence of a need for a coexistence mechanism but just as the absence of stabilization on the specific scale in question. A view of coexistence strictly on local scales would not admit the relevant mechanisms. Source-sink dynamics (Amarasekare and Nisbet 2001) are consistent with the two spatial mechanisms considered here, with the spatial storage effect dominating as dependence of local abundances on local environmental conditions diminishes.

Species sorting is the natural opposite of mass effects in discussions of the local-community concept, where dispersal occurs but is not high enough to overwhelm species interactions on the local scale (Leibold et al. 2004). This outcome is consistent with our results for long environmental correlation lengths, both temporal and spatial, and short dispersal, where fitness-density covariance is the overwhelmingly dominant coexistence mechanism (figs. 4, 6). As figure 1 illustrates, species sorting breaks down to local joint presence as one environment grades into another in space or time. Our results imply that species sorting and mass effects represent ends of a continuum where the dominant coexistence mechanism, on the larger spatial scale of multiple localities, shifts from fitness-density covariance to the spatial storage effect. It is important to emphasize, however, that the spatial storage effect is present along the entire continuum with little change in strength, although its relative importance changes.

Every one of these issues reveals serious limitations in the top-down conception of local communities assembled from species pools. Under the traditional hierarchical concept, environmental filters determine the species able to survive 
at a site. Species interactions further reduce local diversity, although the two cannot be completely separate because the environment determines the outcome of competition (HilleRisLambers et al. 2012; Kraft et al. 2015). Both dispersal and spatiotemporal environmental variation impinge on this metaphor by mixing up the species so that a species that would exclude another no longer does. Population and community dynamics of different locations cannot therefore be treated independently. The regional species pool is best viewed as existing because coexistence of the species is stabilized within the region.

\section{Previous Work on Spatial Coexistence Mechanisms}

Numerous models have addressed spatial coexistence mechanisms in terms of life-history trade-offs (e.g., Hastings 1980; Hanski 1983; Tilman 1994; Amarasekare et al. 2004; Miller and Chesson 2009; Muller-Landau 2010; Samia and Lutscher 2010). In general, they draw a sharp distinction between local interactions and regional coexistence. Although we do not consider life-history trade-offs, they would likely lead to findings that parallel those given here if formulated in a spatially explicit manner and analyzed in terms of scale dependence of mechanisms. For example, continuous relative scales are clearly apparent in the Bolker and Pacala (1999) spatial model of interactions between annual plant species. Models with explicit consideration of environmental variation include that of Miller and Chesson (2009), who find a storage effect arising from disturbance and a resistanceresilience trade-off. Addressing the Janzen-Connell hypothesis, Stump and Chesson (2015) show clear effects of the amount of dispersal on the coexistence of tropical trees. Focusing on spatially periodic environmental change, Samia and Lutscher (2010) and Snyder and Chesson (2003, 2004) find, in agreement with this article, that coexistence depends on the scales of dispersal and environmental change. Similar dependence of coexistence on spatial and temporal scales is found by Aiken and Navarrete (2014), in a model where the necessary numerical and physiological separation is generated by variation in connectivity rather than by local environmental conditions. Finally, Snyder (2008) reveals a strong effect of limited dispersal on the strength of coexistence in an annual plant model for spatially and temporally variable environments. Although not developing the full relationships between coexistence mechanisms, abundance patterns, scales of environmental change and dispersal, and their implications for community concepts, all of this previous work is fully consistent with our conclusions.

\section{Theory of Streams and Advective Habitats}

Variation of stream environments at multiple scales of space and time has long been an organizing principle for under- standing stream diversity (e.g., Vannote et al. 1980; Frissell et al. 1986; Townsend and Hildrew 1994; Poff 1997). More recent work has noted the importance of dispersal for community composition (e.g., Thompson and Townsend 2006; Heino and Mykra 2008; Swan and Brown 2011; Altermatt et al. 2013). Although most work has a strong focus on local community composition, the need to consider the implications of local interactions for broader-scale diversity maintenance has been suggested (Roni et al. 2008; Palmer et al. 2014; Swan and Brown 2014). Streams exhibit complex, multiscaled environmental variation, while the dispersal characteristics linking dynamics at different locations are highly variable in terms of distance, timing, and direction (e.g., Frissell et al. 1986; Hershey et al. 1993; Poff 1997; Peckarsky et al. 2000; Skalski and Gilliam 2000; Hoffman et al. 2006). Any single definition of a local community fails to capture the resulting multiscale nature of community dynamics and diversity maintenance in streams, implying a need for the kind of theory developed here.

A major emphasis has been on biased downstream dispersal, referred to as "drift" or "advection," due to the unidirectional flow of water. Advective dispersal is not limited to streams but is also important along coastlines (Gaines et al. 2003; Byers and Pringle 2006) and in the gut (Ballyk and Smith 1999; O'Brien and Gordon 2011), and it has parallels to climate change (Potapov and Lewis 2004). Our model captures two primary effects of drift: shifted abundance distributions (e.g., Anderson et al. 2005, 2006; Pachepsky et al. 2005) and boundary mortality (washout; e.g., Müller 1982; Speirs and Gurney 2001; Lutscher et al. 2010). We find that spatial shifts in abundance distributions typically have a greater effect on regional coexistence than mortality from washout, unless fitness-density covariance is already weakened by high overall dispersal (fig. 5). In both cases, drift decreases the strength of regional coexistence by weakening fitness-density covariance. As with dispersal in general, the storage effect is relatively insensitive to these changes, continuing to stabilize coexistence even when washout results in negative fitness-density covariance.

Unlike overall dispersal distance, drift has little effect on the degree to which organisms concentrate (fig. 1). Instead, drift weakens fitness-density covariance by shifting concentrations downstream from areas of highest fitness, decreasing the spatial correlation between fitness and density without much effect on the spatial variance of density ("Detailed Examinations of Coexistence Mechanisms" in the appendix). This finding appears, at first, to contradict those of Levine (2003), Lutscher et al. (2007), and Vasilyeva and Lutscher (2012), who found that drift can promote regional coexistence if species have an $r$ - $K$ trade-off and carrying capacity increases monotonically from headwaters to mouth. These assumptions mean that drift shifts the weaker competitor toward areas of higher fitness, a special case of our 
general result that drift affects regional coexistence by shifting populations relative to fitness. Similarly, the results of Aiken and Navarrete (2014) that show coexistence in homogeneous environments with open boundaries and biased dispersal arise because the inferior competitor is better able to concentrate away from areas of dispersal mortality.

Although washout mortality has been a primary concern of drift studies (the drift paradox; e.g., Müller 1982; Speirs and Gurney 2001; Lutscher et al. 2010), we find that washout is important for regional coexistence only when overall dispersal is long-distance and drift proportions are large. In such situations, drift reduces fitness-density covariance because organisms become concentrated downstream in areas with high mortality due to washout. However, there is evidence that organisms have evolved dispersal strategies to avoid washout mortality or reduce the net lifetime drift (Müller 1982; Hershey et al. 1993; Peckarsky et al. 2000; Lowe 2003), making it likely that even the small effects we see here are larger than those in nature. Moreover, drift in streams most commonly occurs within a branched watershed. In such dendritic networks, mortality occurs only at the mouth, while dispersal out of any particular reach simply moves organisms into another, altering local composition (Fagan 2002; Grant et al. 2007; Brown and Swan 2010; Altermatt 2013), suggesting that branching would further reduce the already minor effect of boundaries found here. If the exchanges of individuals between reaches in a dendritic network are large enough to alter dynamics, then the appropriate regional scale is the whole watershed. This fact serves to emphasize the value of viewing species coexistence as a problem of multiple scales in space and time.

\section{Acknowledgments}

This work was supported by National Science Foundation (NSF) grant DEB-1119784 and an NSF Graduate Research Fellowship. We thank J. Bronstein, S. Connolly, Y. Michalakis, M. Rosenzweig, L. Venable, and two anonymous reviewers for helpful comments that improved this article.

\section{Literature Cited}

Aiken, C. M., and S. A. Navarrete. 2014. Coexistence of competitors in marine metacommunities: environmental variability, edge effects, and the dispersal niche. Ecology 95:2289-2302.

Altermatt, F. 2013. Diversity in riverine metacommunities: a network perspective. Aquatic Ecology 47:365-377.

Altermatt, F., M. Seymour, and N. Martinez. 2013. River network properties shape $\alpha$-diversity and community similarity patterns of aquatic insect communities across major drainage basins. Journal of Biogeography 40:2249-2260.

Amarasekare, P., M. Hoopes, N. Mouquet, and M. Holyoak. 2004 Mechanisms of coexistence in competitive metacommunities. American Naturalist 164:310-326.
Amarasekare, P., and R. M. Nisbet. 2001. Spatial heterogeneity, sourcesink dynamics, and the local coexistence of competing species. American Naturalist 158:572-584

Anderson, K. E., R. M. Nisbet, and S. Diehl. 2006. Spatial scaling of consumer-resource interactions in advection-dominated systems. American Naturalist 168:358-372.

Anderson, K. E., R. M. Nisbet, S. Diehl, and S. Cooper. 2005. Scaling population responses to spatial environmental variability in advectiondominated systems. Ecology Letters 8:933-943.

Auerbach, D. A., and N. L. Poff. 2011. Spatiotemporal controls of simulated metacommunity dynamics in dendritic networks. Lournal of the North American Benthological Society 30:235-251.

Ballyk, M., and H. Smith. 1999. A model of microbial growth in a plug flow reactor with wall attachment. Mathematical Biosciences 158:95-126.

Bolker, B. M., and S. Pacala. 1999. Spatial moment equations for plant competition: understanding spatial strategies and the advantages of short dispersal. American Naturalist 153:575-602.

Brown, B. L., and C. M. Swan. 2010. Dendritic network structure constrains metacommunity properties in riverine ecosystems. Journal of Animal Ecology 79:571-580.

Byers, J. E., and J. M. Pringle. 2006. Going against the flow: retention, range limits and invasions in advective environments. $\mathrm{Ma}-$ rine Ecology Progress Series 313:27-41.

Caswell, H., and J. Cohen. 1991. Disturbance, interspecific interaction and diversity in metapopulations. Biological Journal of the Linnean Society 42:193-218.

. 1995. Red, white, and blue: environmental variance spectra and coexistence in metapopulations. Iournal of Theoretical Biology 176:301-316.

Chesson, P. 1985. Coexistence of competitors in spatially and temporally varying environments: a look at the combined effects of different sorts of variability. Theoretical Population Biology 28: 263-287.

1994. Multispecies competition in variable environments. Theoretical Population Biology 45:227-276.

2000a. General theory of competitive coexistence in spatiallyvarying environments. Theoretical Population Biology 58:211-237.

- 2000b. Mechanisms of maintenance of species diversity. Annual Review of Ecology and Systematics 31:343-366.

. 2003. Quantifying and testing coexistence mechanisms arising from recruitment fluctuations. Theoretical Population Biology 64:345-357.

-2008. Quantifying and testing species coexistence mechanisms. Pages 119-164 in E. B. Valladares, A. Camacho, A. Elosegi, C. Gracia, M. Estrada, J. C. Senar, and J. M. Gili, eds. Unity in diversity: reflections on ecology after the legacy of Ramon Margalef. Fundación BBVA, Bilbao, Spain.

. 2012. Scale transition theory: its aims, motivations and predictions. Ecological Complexity 10:52-68.

Chesson, P., M. Donahue, B. A. Melbourne, and A. L. W. Sears 2005. Scale transition theory for understanding mechanisms in metacommunities. Pages 279-306 in M. Holyoak, M. A. Leibold, and R. D. Holt, eds. Metacommunities. University of Chicago Press, Chicago.

Chesson, P., and S. Ellner. 1989. Invasibility and stochastic boundedness in monotonic competition models. Journal of Mathematical Biology 27:117-138.

Connell, J. H. 1978. Diversity in tropical rain forests and coral reefs. Science 199:1302-1310. 
Cornell, H. V., and S. P. Harrison. 2014. What are species pools and when are they important? Annual Review of Ecology, Evolution and Systematics 45:45-67.

Cottenie, K. 2005. Integrating environmental and spatial processes in ecological community dynamics. Ecology Letters 8:1175-1182.

Fagan, W. F. 2002. Connectivity, fragmentation, and extinction risk in dendritic metapopulations. Ecology 83:3243-3249.

Frissell, C. A., W. J. Liss, C. E. Warren, and M. D. Hurley. 1986. A hierarchical framework for stream habitat classification: viewing streams in a watershed context. Environmental Management 10: 199-214.

Gaines, S. D., B. Gaylord, and J. L. Largier. 2003. Avoiding current oversights in marine reserve design. Ecological Applications 13: $32-46$.

Gilbert, B., and M. J. Lechowicz. 2004. Neutrality, niches, and dispersal in a temperate forest understory. Proceedings of the National Academy of Sciences of the USA 101:7651-7656.

Grant, E. H. C., W. H. Lowe, and W. F. Fagan. 2007. Living in the branches: population dynamics and ecological processes in dendritic networks. Ecology Letters 10:165-175.

Hanski, I. 1983. Coexistence of competitors in patchy environment. Ecology 64:493-500.

Hastings, A. 1980. Disturbance, coexistence, history, and competition for space. Theoretical Population Biology 18:363-373.

Heino, J., and H. Mykra. 2008. Control of stream insect assemblages: roles of spatial configuration and local environmental factors. Ecological Entomology 33:614-622.

Hershey, A. E., J. Pastor, B. J. Peterson, and G. W. Kling. 1993. Stable isotopes resolve the drift paradox for Baetis mayflies in an Arctic river. Ecology 74:2315-2325.

HilleRisLambers, J., P. B. Adler, J. M. Levine, and M. M. Mayfield. 2012. Rethinking community assembly through the lens of coexistence theory. Annual Review of Ecology, Evolution, and Systematics 43:227-248.

Hoffman, A. L., J. D. Olden, J. B. Monroe, N. L. Poff, T. Wellnitz, and J. A. Wiens. 2006. Current velocity and habitat patchiness shape stream herbivore movement. Oikos 115:358-368.

Hutchinson, G. 1951. Copepodology for the ornithologist. Ecology 32:571-577.

Janke, W. 1996. Monte Carlo simulations of spin systems. Pages 1043 in K. H. Hoffman and M. Schreiber, eds. Computational physics. Springer, Berlin.

Kraft, N., P. B. Adler, O. Godoy, and E. C. James. 2015. Community assembly, coexistence and the environmental filtering metaphor Functional Ecology 29:592-599.

Kuglerová, L., R. Jansson, R. A. Sponseller, H. Laudon, and B. MalmRenöfält. 2015. Local and regional processes determine plant species richness in a river-network metacommunity. Ecology 96:381391.

Lake, P. 2000. Disturbance, patchiness, and diversity in streams. Lournal of the North American Benthological Society 19:573-592.

Lavorel, S., and P. Chesson. 1995. How species with different regeneration niches coexist in patchy habitats with local disturbances. Oikos 74:103-114.

Leibold, M. A., M. Holyoak, N. Mouquet, P. Amarasekare, J. M. Chase, M. Hoopes, R. D. Holt, et al. 2004. The metacommunity concept: a framework for multi-scale community ecology. Ecology Letters 7:601-613.

Levin, S. A. 1976. Population dynamics models in heterogeneous environments. Annual Review of Ecology and Systematics 7:287-310
Levine, J. M. 2003. A patch modeling approach to the communitylevel consequences of directional dispersal. Ecology 84:1215-1224.

Levins, R. 1969. Some demographic and genetic consequences of environmental heterogeneity for biological control. Bulletin of the Entomological Societv of America 237-240.

Lowe, W. H. 2003. Linking dispersal to local population dynamics: a case study using a headwater salamander system. Ecology 84:2145-2154.

Lutscher, F., E. McCauley, and M. A. Lewis. 2007. Spatial patterns and coexistence mechanisms in systems with unidirectional flow. Theoretical Population Biology 71:267-277.

Lutscher, F., R. M. Nisbet, and E. Pachepsky. 2010. Population persistence in the face of advection. Theoretical Ecology 3:271-284.

Miller, A. D., and P. Chesson. 2009. Coexistence in disturbance-prone communities: how a resistance-resilience trade-off generates coexistence via the storage effect. American Naturalist 173:E30-E43.

Moloney, K. A., S. A. Levin, N. R. Chiariello, and L. Buttel. 1992. Pattern and scale in a serpentine grassland. Theoretical Population Biology 41:257-276.

Müller, K. 1954. Investigation on the organic drift in North Swedish streams. Institute of Freshwater Research Drottningholm Report 35: 133-148.

- 1982. The colonization cycle of freshwater insects. Oecologia (Berlin) 52:202-207.

Muller-Landau, H. C. 2010. The tolerance-fecundity trade-off and the maintenance of diversity in seed size. Proceedings of the National Academy of Sciences of the USA 107:4242-4247.

Mykra, H., J. Heino, and T. Muotka. 2004. Variability of lotic macroinvertebrate assemblages and stream habitat characteristics across hierarchical landscape classifications. Environmental Management 34:341-352.

O'Brien, C. L., and D. M. Gordon. 2011. Effect of diet and gut dynamics on the establishment and persistence of Escherichia coli. Microbiology 157:1375-1384.

Ovaskainen, O. 2002. The effective size of a metapopulation living in a heterogeneous patch network. American Naturalist 160:612-628.

Pacala, S. W., C. D. Canham, J. Saponara, J. A. Silander, Jr., R. K. Kobe, and E. Ribbens. 1996. Forest models defined by field measurements: estimation, error analysis and dynamics. Ecological Monographs 66:1-43.

Pachepsky, E., F. Lutscher, R. M. Nisbet, and M. A. Lewis. 2005. Persistence, spread and the drift paradox. Theoretical Population Biology 67:61-73.

Palmer, M. A., K. L. Hondula, and B. J. Koch. 2014. Ecological restoration of streams and rivers: shifting strategies and shifting goals. Annual Review of Ecology, Evolution, and Systematics 45:247-269.

Peckarsky, B., B. Taylor, and C. Caudill. 2000. Hydrologic and behavioral constraints on oviposition of stream insects: implications for adult dispersal. Oecologia (Berlin) 125:186-200.

Poff, N. L. 1997. Landscape filters and species traits: towards mechanistic understanding and prediction in stream ecology. Lournal of the North American Benthological Society 16:391-409.

Potapov, A. B., and M. A. Lewis. 2004. Climate and competition: the effect of moving range boundaries on habitat invasibility. Bulletin of Mathematical Biology 66:975-1008.

Pulliam, H. 1988. Sources, sinks, and population regulation. American Naturalist 132:652-661.

Ricklefs, R. E. 2008. Disintegration of the ecological community. American Naturalist 172:741-750.

Roni, P., K. Hanson, and T. J. Beechie. 2008. Global review of the physical and biological effectiveness of stream habitat rehabilita- 
tion techniques. North American Journal of Fisheries Managemen 28:856-890.

Samia, Y., and F. Lutscher. 2010. Coexistence and spread of competitors in heterogeneous landscapes. Bulletin of Mathematical Biology 72:2089-2112.

Schreiber, S. J. 2012. Persistence for stochastic difference equations: a mini-review. Iournal of Difference Equations and Applications 18:1381-1403.

Shmida, A., and S. Ellner. 1984. Coexistence of plant species with similar niches. Vegetatio 58:29-55.

Skalski, G. T., and J. F. Gilliam. 2000. Modeling diffusive spread in a heterogeneous population: a movement study with stream fish. Ecology 81:1685-1700.

Slatkin, M. 1974. Competition and regional coexistence. Ecology $55: 128-134$.

Snyder, R. E. 2008. When does environmental variation most influence species coexistence? Theoretical Ecology 1:129-139.

Snyder, R. E., and P. Chesson. 2003. Local dispersal can facilitate coexistence in the presence of permanent spatial heterogeneity. Ecology Letters 6:301-309.

. 2004. How the spatial scales of dispersal, competition, and environmental heterogeneity interact to affect coexistence. American Naturalist 164:633-650.

Sousa, W. 1979. Disturbance in marine intertidal boulder fields: the nonequilibrium maintenance of species diversity. Ecology 60:1225-1239.

Speirs, D., and W. Gurney. 2001. Population persistence in rivers and estuaries. Ecology 82:1219-1237.

Stump, S. M., and P. Chesson. 2015. Distance-responsive predation is not necessary for the Janzen-Connell hypothesis. Theoretical Population Biology 106:60-70.
Swan, C. M., and B. L. Brown. 2011. Advancing theory of community assembly in spatially structured environments: local vs regional processes in river networks. Journal of the North American Benthological Society 30:232-234.

. 2014. Using rarity to infer how dendritic network structure shapes biodiversity in riverine communities. Ecography 37:993-1001. Thompson, R., and C. R. Townsend. 2006. A truce with neutral theory: local deterministic factors, species traits and dispersal limitation together determine patterns of diversity in stream invertebrates. Journal of Animal Ecology 75:476-484.

Tilman, D. 1994. Competition and biodiversity in spatially structured habitats. Ecology 75:2-16.

Townsend, C. R. 1989. The patch dynamics concept of stream community ecology. Journal of the North American Benthological Society 8:36-50.

Townsend, C. R., and A. G. Hildrew. 1994. Species traits in relation to a habitat templet for river systems. Freshwater Biology 31:265-275.

Turelli, M. 1978. Does environmental variability limit niche overlap? Proceedings of the National Academy of Sciences of the USA 75:5085-5089.

Vannote, R. L., G. W. Minshall, K. W. Cummins, J. R. Sedell, and C. E. Cushing. 1980. The river continuum concept. Canadian Journal of Fisheries and Aquatic Sciences 37:130-137.

Vasilyeva, O., and F. Lutscher. 2012. How flow speed alters competitive outcome in advective environments. Bulletin of Mathematical Biology 74:2935-2958.

Associate Editor: Sean R. Connolly Editor: Yannis Michalakis

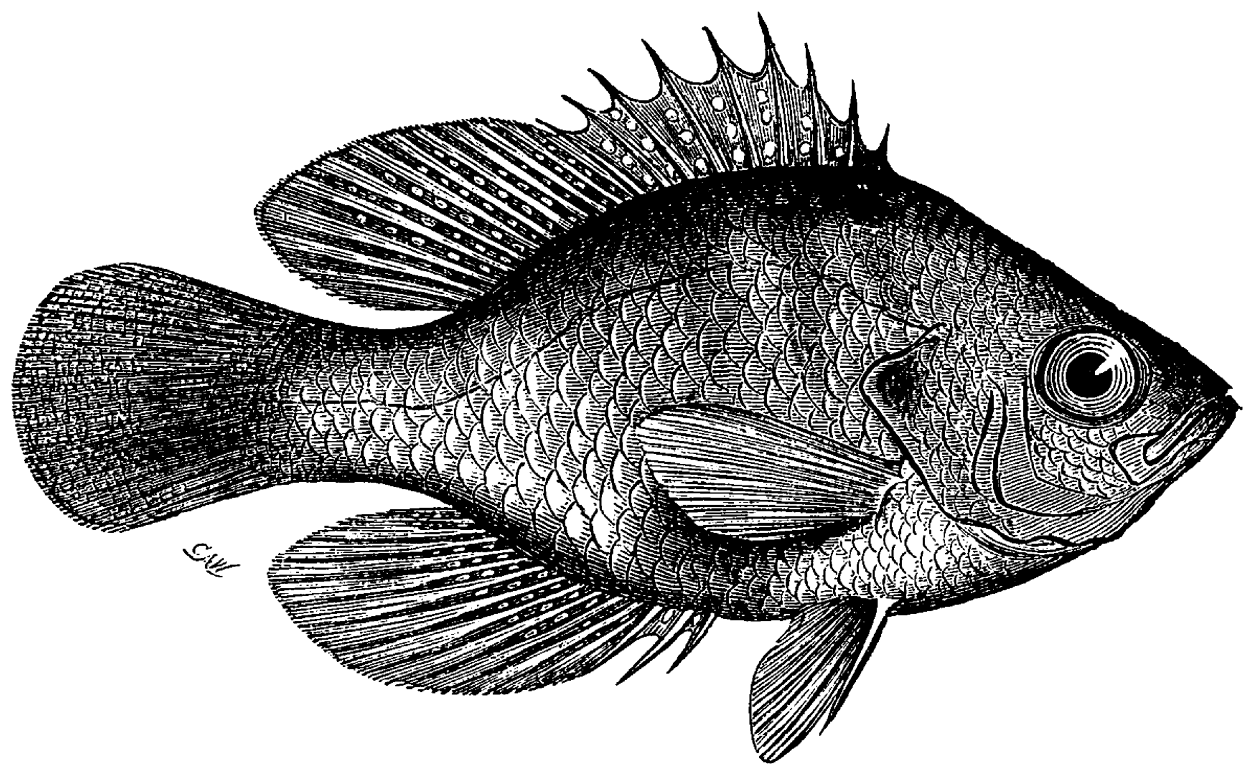

"Spotted Sun-fish (Enneacanthus guttatus)... The main interest attaching to this species, at least to us, is the fact of its occupying many small, sluggish streams, similar and side by side with others that harbor, though less abundantly, the E. obsesus. We never yet have found them associated in small streams, in the tributaries of the river; yet, in the Delaware itself the E. obesus is occasionally, and the guttatus frequently found." From "Mud-Loving Fishes" by Charles C. Abbott (The American Naturalist 1870, 4:385-391). 